\title{
An Experimental Study of FSO Link Performance in Desert Environment
}

\author{
Maged Abdullah Esmail, Student Member, IEEE, Habib Fathallah, Senior Member, IEEE, \\ and Mohamed-Slim Alouini, Fellow, IEEE
}

\begin{abstract}
Free space optical (FSO) communication systems are affected by dust particles suspended in the atmosphere in arid and semi-arid regions. The presence of these particles in the air severely affects the optical link, reduces its availability and causes service outage. In the literature, the effect of dust on the microwave signals has been widely investigated. However, for FSO communication systems that exploit shorter wavelengths, information and research is still very limited yet almost inexistent. Therefore, in this paper, we investigate the performance of FSO links under dust storms. We designed a chamber to emulate this specific environment and carry out measurements. From the experimental investigations, we derive and propose an empirical model for the signal attenuation as a function of the visibility range. The results show acceptable performance for FSO links, under moderate and light dust, with potential reach distance of hundreds of meters to few kilometers. Furthermore, a comparison analysis shows that the dust induces 7 times higher attenuation than fog.
\end{abstract}

Index Terms-free space optics, dust storm, visibility range, attenuation model.

\section{INTRODUCTION}

$\mathbf{O}$ PTICAL wireless communication, also known as free space optical (FSO) communication, is a promising technology to solve some inherent issues in radio frequency (RF) technology such as last mile bottleneck in $4 \mathrm{G} / 5 \mathrm{G}$ wireless networks. Theoretically, it has unlimited and unlicensed bandwidth that makes it an attractive solution with low potential cost. Commercial products are available today with data rates up to $10 \mathrm{Gbps}$. In addition, it has other advantages such as ease and fast installation, immunity to interference, security, etc. Because of theses interesting properties, FSO systems have found many applications such as $4 \mathrm{G} / 5 \mathrm{G}$ backhauling, cooperative private networks, disaster recovery, defense and national security, temporary and special events, etc. [1].

Most of the studies on FSO system were performed in Europe, East Asia, and North America where the main conditions are fog, rain, snow, and turbulence. In these studies, the effect of dust is usually ignored mainly because it does not exist in the studied environments. Fog has been identified as the one that causes most of the attenuation, because it has particle size that is close to the signal wavelength [1].

M. A. Esmail and H. Fathallah are with the Electrical Engineering Department, King Saud University, Riyadh 11421, Saudi Arabia. They are also with the KACST Technology Innovation Center in Radio Frequency and Photonics (RFTONICs) center, Riyadh 11421, Saudi Arabia. e-mail: (mesmail@ksu.edu.sa).

M.-S. Alouini is with the Computer, Electrical, and Mathematical Science and Engineering (CEMSE) Division, King Abdullah University of Science and Technology (KAUST), Thuwal, Makkah Province, Saudi Arabia.

Manuscript received xxx xx, xxxx; revised xxx xx, xxx.
In this paper, we focus on arid and semi-arid areas, where dust is the main impairment for FSO systems. These areas are subject to frequent dust storms, where fine dust particles may be lifted as high as $1-3 \mathrm{~km}$ into the atmosphere and last for few days after dust storm [2]. Moreover, we aim to compare the attenuation created by fog and that by dust. Worthy to mention that the size of fog particles is comparable to that of dust, while their compositions are different.

For microwave and millimeter wave (MMW), there are some field measurements, and proposed models, for signal attenuation. In general, it is found that the attenuation of microwave and MMW signal by dust is not a serious problem except under very dense storms [3]. Investigation, modelling and research of the dusty channel in FSO links is still in its infancy. Very limited effort has been spent in this direction. To the best of our knowledge, there is no channel attenuation model proposed or developed for such environment. In desert environment, the dust effect will be paramount for any future installation of FSO links in current and next generation of communication networks. It is straightforward that the prediction of attenuation, caused by dust storms, will be a serious challenge for the establishment of an FSO communication link and network.

Because of the difficulty to predict the occurrence of specific atmospheric effects like fog, rain, smoke, and dust events, and because of the difficulty to repeat the measurements under the same conditions, the authors in [4-7] made a pioneering but preliminary investigation of the FSO performance using controlled environment (chamber). In particular, the authors in [4] studied the FSO performance using on-off keying (OOK) modulation and pulse position modulation (PPM) schemes. The results show that PPM has better performance. In [5], the authors compared the performance of $\mathrm{THz}$ wave and $1550 \mathrm{~nm}$ infrared FSO signal. The results show two orders of magnitude higher attenuation for infrared than $\mathrm{THz}$ link, which is quite expected mainly because FSO link has a wavelength close to the diameter of the dust particles. In this paper, we also build a laboratory chamber to investigate the performance of the FSO communication system under dust conditions. The chamber emulates the outdoor dusty environment and enables to measure the signal attenuation as a function of the visibility range. An empirical model is developed based on these measurements. Our results show poor performance for FSO link under dense dust. Under moderate dust, a communication link can be established with a length of hundred meters similar to the cell size of $5 \mathrm{G}$ wireless networks. Under light dust, the FSO link can be extended to up few kilometers. In general, we 
observed that dust introduces about 7 times higher attenuation than fog for the same visibility values.

The remaining of the paper is organized as follows. In Section II we review the dust characterization and its relation to the visibility range. In Section III, we discuss our experimental setup. The obtained results are presented in Section IV. Finally, we summarize our conclusions in Section V.

\section{DUST CHARACTERISTICS}

In FSO, the signal wavelength is chosen to operate in the low absorption bands. Hence, absorption contribution to the total attenuation coefficient becomes very small when compared to scattering effect [8]. Signal scattering occurs due to different types of conditions such as fog, rain, smoke, dust, snow, etc. Investigating the effect of these conditions on the FSO signal can be achieved using one of two available methods. The first method uses theoretical based theorems such as Mie theory. However, theoretical approaches require some parameters that may not be available at the installation location such as particle size, particle distribution, refractive index, etc. [6].

The second method for signal attenuation calculation which is widely used in literature for practical applications depends on experimental observation [6]. In this method, empirical models are developed using the visibility range data to characterize the channel. Visibility data can be obtained easily form meteorological stations located close to the installation location. Visibility is defined as the distance to an object where the image distinction drops to a certain percentage of what would be if the object were nearby instead [6]. Image distinction that drops to $2 \%$ and $5 \%$ is used to be considered in visibility definition. The visibility is measured at $550 \mathrm{~nm}$ which represents the maximum intensity of solar spectrum. Visibility is considered as a measure of the severity of the dust storm. Low visibility implies high concentration of dust particles in the atmosphere.

Based on visibility range, dust events are divided into four categories according to World Meteorological Organization (WMO) classification as listed in Table I [9]. The first type is dust haze (light dust) where wide spread dust float up and become suspended homogeneously in the air. This event occurs due to dust storm that happens at a considerable distance from the observation location. The visibility related to this event is around $10 \mathrm{~km}$ or less. Blowing dust (light dust) is the second type in which dust particles are blown in the observation location by winds and the visibility reduces between $1 \mathrm{~km}$ and $10 \mathrm{~km}$. When the visibility reduces to $0.2 \mathrm{~km}$ due to strong winds that blow more dust particles, the event is called dust storm (moderate dust). Finally, severe dust storm (dense dust) occurs when the visibility becomes less than $0.2 \mathrm{~km}$ where large quantity of dust particles is lifted up by very strong winds. The visibility range can be determined from the signal attenuation coefficient $\alpha$ and the visual threshold $T_{t h}$ by [6]

$$
V=-\frac{10 \log T_{t h}}{\alpha}, \quad(\mathrm{km})
$$

TABLE I

Dust Event Classification Based on Visibility Range

\begin{tabular}{lcccc}
\hline \hline Dust Type & $\begin{array}{c}\text { Severe } \\
\text { dust storm }\end{array}$ & Dust storm & Blowing dust & Dust haze \\
\hline \hline Description & Dense & Moderate & Light & Light \\
$\mathrm{V}(\mathrm{km})$ & $<0.2$ & $0.2-1$ & $1-10$ & $\leq 10$ \\
\hline
\end{tabular}

where $T_{t h}=0.02$ according to Koschmieder law. The signal attenuation coefficient is given by [6]

$$
\alpha=-\frac{10 \log T}{4.343 L}, \quad(\mathrm{~dB} / \mathrm{km})
$$

where $T$ is the transmittance of the optical signal at $550 \mathrm{~nm}$ and $L$ is the link length.

\section{EXPERIMENT DESCRIPTION}

Investigating outdoor FSO communication is difficult due to the long time it takes. Therefore, controlled environment has been used to emulate the outdoor impairments. Using a controlled environment, the atmospheric conditions can be reproduced to perform different measurements under the same conditions [4-7]. Fig. 1(a) shows our FSO link setup including the chamber design. The dusty channel is represented by a chamber of $90 \times 40 \times 40 \mathrm{~cm}^{3}$ size. The amount of dust introduced to the chamber is controlled by fans which blow up the dust particles and create homogeneous environment. Uncoated high precise transparent windows with $4 \%$ typical loss per surface are used to allow passing signals at different wavelengths.

In the transmitter side, two single mode fiber (SMF) coupled laser diodes (LDs) are used. The infrared Keysight N7714A tunable LD is used to generate a continuous signal at $1550 \mathrm{~nm}$ wavelength with $4 \mathrm{~mW}$ output power. A collimator that exploits an aspheric lens pre-aligned at $1550 \mathrm{~nm}$ is used to transmit the LD's signal over free space. After passing the dusty chamber, the transmitted light hits a photodiode optical power sensor (Thorlabs S122C) having $-43 \mathrm{dBm}$ sensitivity, that is connected to a power meter benchtop console (Thorlabs PM320E). Finally, a data acquisition system is used to collect the data and store it for offline processing.

To measure the visibility, another link using $520 \mathrm{~nm}$ wavelength parallel to the $1550 \mathrm{~nm}$ link is used. This link uses Fabry-Perot LD (Thorlabs S3FC520) with $2 \mathrm{~mW}$ output power. Similar to the $1550 \mathrm{~nm}$ link, the $550 \mathrm{~nm}$ link uses aspheric lens collimator pre-aligned at $532 \mathrm{~nm}$ for light transmission over free space and a photodiode (Thorlabs S120C) with $-43 \mathrm{dBm}$ sensitivity in the receiver side. Note that, in FSO communication systems, the $1550 \mathrm{~nm}$ wavelength is used because it has lower absorption than other bands in addition to better eye safety, allowing the transmission of higher power levels [8]. Furthermore, this wavelength is compatible with the current fiber based infrastructure.

To compensate for dust sticks on the transparent windows, we injected some dust to the chamber and blown it several times. This allows dust to stick to the walls and windows reaching a kind of saturation sticking level. We started the measurements by injecting the dust into the chamber and 


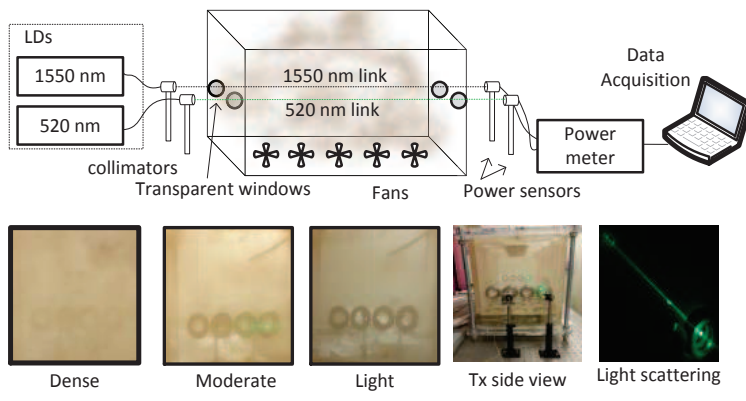

(a)

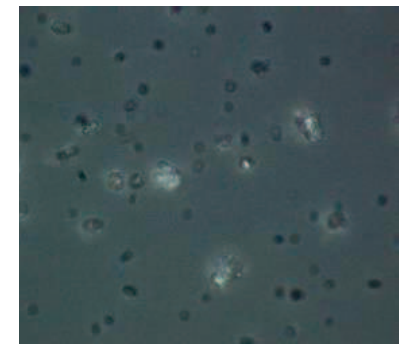

(b)

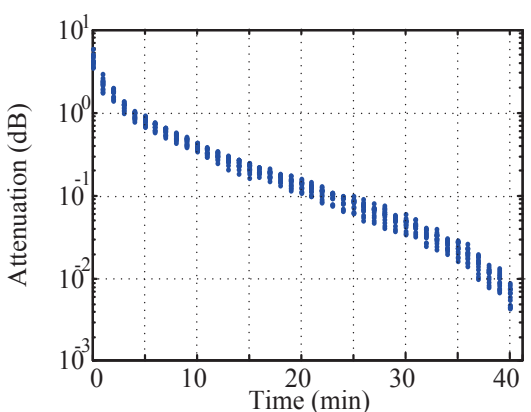

(c)

Fig. 1. (a) Experimental setup to measure dust effect on FSO links, (b) Dust particles viewed using optical microscopy, (c) Attenuation (dB) versus time.

measure the received signal automatically every $0.1 \mathrm{sec}$ using the acquisition system. Then we took the average over $1 \mathrm{~min}$. The measurements continued till all dust particles fall down. The visibility was calculated from the $520 \mathrm{~nm}$ link using (1) and (2) where $T$ is found as the ratio of received power when there is dust to that when there is no dust. For each measurement, we allowed one minute for the dust to settle down before taking measurements. The experiment was carried out in dark room to eliminate the ambient light noise.

\section{RESUlTS AND Discussions}

We started the experimental work by filling the chamber with a quantity of dust. The dust sample was collected from a real dust storm event that occurred in Riyadh city. The average particle size is $4 \mu \mathrm{m}$ measured using an optical microscopy image. Fig. 1(b) shows a microscopy image of the dust with $100 \times$ magnification. Data acquisition continued for 40 minutes until the transmission space becomes clear of dust and the received power increases back to its initial level before the dust injection. The experiment was repeated 15 times under the same conditions. To measure the signal attenuation using the $1550 \mathrm{~nm}$ wavelength $\mathrm{LD}$, the received signal power in $\mathrm{dBm}$ was subtracted from the transmitted power when there was no dust. Simultaneously, we measure the visibility range using the $520 \mathrm{~nm} \mathrm{LD}$.

\section{A. Signal Attenuation}

Fig. 1(c) shows the signal attenuation versus time for the set of 15 repeated measurements. The maximum signal attenuation was $7 \mathrm{~dB}$. The measurements' divergence is small reflecting the setup capability to reproduce the similar results under the similar conditions. In Fig. 2(a), we calculated the specific attenuation in $\mathrm{dB} / \mathrm{km}$ versus the visibility range. For very low visibility corresponding to dense dust $(V<0.2 \mathrm{~km})$, the obtained attenuation is about $300 \mathrm{~dB} / \mathrm{km}$. For moderate dust $(0.2 \mathrm{~km}<V<1 \mathrm{~km})$, the corresponding signal attenuation is between $300 \mathrm{~dB} / \mathrm{km}$ at $V=0.2 \mathrm{~km}$ and $50 \mathrm{~dB} / \mathrm{km}$ at $V=1 \mathrm{~km}$. As the visibility range increases more than $1 \mathrm{~km}$ (light dust), the signal attenuation substantially reduces. As an example, we measure $10 \mathrm{~dB} / \mathrm{km}$ at $4 \mathrm{~km}$ visibility.

The measurements in Fig. 2(a) show reciprocal relationship between the signal attenuation and the visibility. Using non- linear least square regression, a model for signal attenuation as a function of the visibility range is obtained as

$$
\alpha=-k \times V^{b}, \quad(\mathrm{~dB} / \mathrm{km})
$$

where $V$ is given in $\mathrm{km}$, and the coefficients are found to be $k=52$, and $b=-1.05$. Fig. 2(a) shows the fitted curve with the measured data. To analyze the reliability of this model, we calculated the goodness of fit test using Rsquare measure, the confidence bounds, and the root mean square error (RMSE) between the obtained model and the measured data. The R-square measure is found to be 0.99 which shows good fitting. The confidence bounds with $95 \%$ level of certainty are found to be: $k=52(50.94,53.25)$ and $b=-1.05(-1.056,-1.037)$ where both intervals have narrow width indicating high precision parameters estimation. For the RMSE, we found $17 \mathrm{~dB}$ RMSE between the obtained model and the measurements over the whole range of visibility shown in Fig. 2(a).

\section{B. Effect of Link Length}

The performance of the FSO system in dusty channel as a function of the visibility range and the link distance is shown in Fig. 2(b). The receiver sensitivity corresponding to a system with $2.5 \mathrm{Gbps}$ data rate is considered to be $P_{s}=-34 \mathrm{dBm}$. For $P_{t}=22 \mathrm{dBm}$, we achieve a link with $1 \mathrm{~km}$ length under light dust $(V>1 \mathrm{~km})$. For moderate dust storm which has visibility between $0.2 \mathrm{~km}$ and $1 \mathrm{~km}$, the link length is reduced to $200 \mathrm{~m}$ at $V=200 \mathrm{~m}$. Under severe dust storm which has dense dust, the visibility becomes lower than $0.2 \mathrm{~km}$ leading to short link length less than $200 \mathrm{~m}$.

Fig. 2(b) also shows the effect of increasing the transmitted power. We notice that under low visibility, power increment dose not lead to noticeable improvement in system performance. However, under high visibility and under light dust, the power increment leads to a good improvement in the system performance.

The obtained results show that FSO is a short range technology in arid regions where dust limits its reach. Under moderate dust, only few hundred meters are possible in a single span link before requiring amplification for a subsequent span for equivalent length. Note that the trend in next generation wireless networks (5G) is to use small size cells with few 


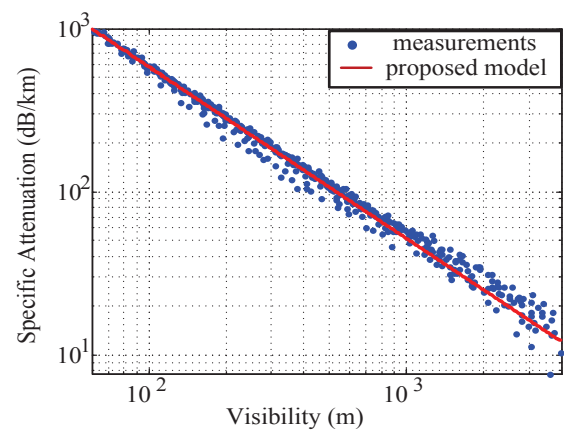

(a)

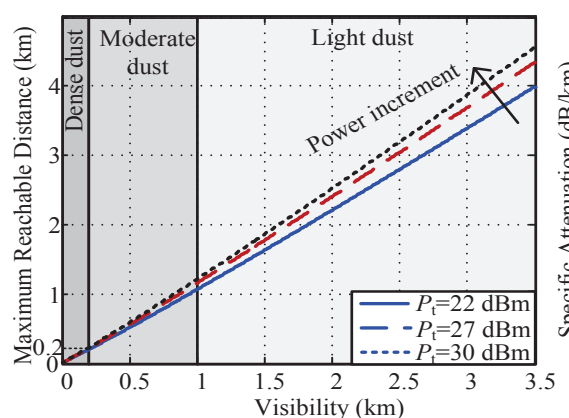

(b)

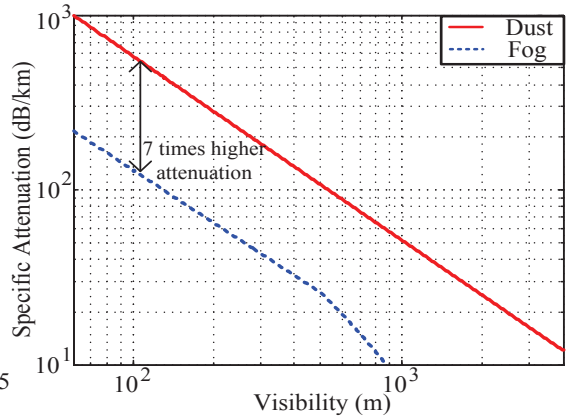

(c)

Fig. 2. (a) Specific attenuation in $\mathrm{dB} / \mathrm{km}$ versus the visibility range, (b) Effect of link length on signal attenuation with $P_{s}=-34 \mathrm{dBm}$, (c) Comparison of signal attenuation due to dust and fog.

hundred meters diameter. With this small size cells, we can see FSO as a good point-to-point or backhauling communication link.

Furthermore, the results tell us that under dense dust with $V<0.2 \mathrm{~km}$, the link reach is further reduced to less than $200 \mathrm{~m}$ which may introduce service outage. Fortunately, the occurrence of dense dust storm is rare and spans for only few hours per year. A study of dust storms occurred in Riyadh city for ten years from 1972 to 1982 showed that visibility reduction less than $0.2 \mathrm{~km}$ represents $1.61 \times 10^{-3}$ time of the year [10]. This means for $99.83 \%$ of the year time, the visibility is higher than $0.2 \mathrm{~km}$.

\section{Comparsion of Dust and Fog Attenuation}

In the literature, Fog is used to be classified as the extreme limitation for FSO performance. This is because the particle size of fog is close to the signal wavelength used in FSO communications. This consideration of signal attenuation in fog is obtained from field measurements that were performed mainly in Europe and East Asia, which do not have arid desert climate. Recall that, to our best knowledge, there are no field measurements on FSO system performance in desert environment. Therefore, fog was considered as the main challenge for FSO systems. In order to compare the behavior of FSO links under dust and fog, we compared our obtained results with Kim model of signal attenuation in fog [6] which is given by

$$
\alpha=\frac{13}{V}\left(\frac{\lambda}{550}\right)^{-q}, \quad(\mathrm{~dB} / \mathrm{km})
$$

where $V$ is the visibility range in $\mathrm{km}, \lambda$ is the signal wavelength in $\mu \mathrm{m}$, and the coefficient $q$ has the following values:

$$
q=\left\{\begin{array}{lr}
0, & V<0.5 \mathrm{~km} \\
V-0.5, & 0.5 \mathrm{~km}<V<1 \mathrm{~km} \\
0.16 V+0.34, & 1 \mathrm{~km}<V<6 \mathrm{~km} \\
1.3, & 6 \mathrm{~km}<V<50 \mathrm{~km} \\
1.6, & 50 \mathrm{~km}<V
\end{array}\right.
$$

Fig. 2(c) shows that the FSO signal undergoes much more attenuation in dusty weather. This attenuation is about 7 times higher than that of fog. Note that both particles of fog and dust have a size that is close to the FSO signal wavelength. However, the fog particles contain water resulting in less scattering for the penetrating signal.

\section{CONCLUSION}

In this paper, we investigated the performance of FSO communication system in dusty environment. We designed a controlled environment chamber for this purpose. Our results showed possibility of establishing short FSO links under moderate and light dust. However, dense dusty conditions can affect significantly the performance of FSO links. In addition, we found that dust introduces about 7 time signal attenuation higher than fog at similar visibility values.

\section{REFERENCES}

[1] M. Khalighi and M. Uysal, "Survey on free space optical communication: A communication theory perspective," IEEE Cотmu. Surveys and Tutorials, vol. 16, no. 4, pp. 2231-2258, June 2014.

[2] N. Oanh, Integrated Air Quality Management: Asian Case Studies: CRC Press, 2012.

[3] A. Musa, S. O. Bashir, and A. H. Abdalla, "Review and assessment of electromagnetic wave propagation in sand and dust storms at microwave and millimeter wave bands - Part II," Progress In Electromagnetics Research M, vol. 40, pp. 101-110, 2014.

[4] Z. Ghassemlooy, J. Perez, and E. Leitgeb, "On the performance of FSO communications links under sandstorm conditions," in 12th Int. Con. on Telec., Zagreb, June 2013, pp. 53-58.

[5] K. Su, L. Moeller, R. Barat, and J. Federici, "Experimental comparison of terahertz and infrared data signal attenuation in dust clouds," J. Opt. Soc. Am. A, vol. 29, no. 11, pp. 2360-2366, 2012.

[6] M. Ijaz, Z. Ghassemlooy, J. Pesek, O. Fiser, H. Minh, and E. Bentley, "Modeling of Fog and smoke attenuation in free space optical communications link under controlled laboratory conditions," IEEE Jour. of Lightwave Techn., vol. 31, no. 11, pp. 1720 1726, June 2013.

[7] S. Rajbhandari, Z. Ghassemlooy, P. Haigh, T. Kanesan and X. Tang, "Experimental Error Performance of Modulation Schemes Under a Controlled Laboratory Turbulence FSO Channel," Jour. of Lightwave Tech., vol. 33, no. 1, pp. 244-250, Jan. 2015.

[8] I. I. Kim, B. McArthur, and E. J. Korevaar, "Comparison of laser beam propagation at $785 \mathrm{~nm}$ and $1550 \mathrm{~nm}$ in fog and haze for optical wireless communications," in Proc. SPIE 4214, Optical Wireless Communications III, 2001, pp. 26-37.

[9] Y. Shao, Physics and Modeling of Wind Erosion, 2nd ed. vol. 37: Springer Science and Business Media, 2008.

[10] M. A. Alhaider, "Radio wave propagation into sandstorms-system design based on ten-years visibility data in Riyadh, Saudi Arabia," Inter. Jour. of Infr. and Millimeter Waves, vol. 7, no. 9, pp. 1339-1359, Sept. 1986. 